PROCEEDINGS OF THE

AMERICAN MATHEMATICAL SOCIETY

Volume 137, Number 2, February 2009, Pages 401-408

S 0002-9939(08)09587-7

Article electronically published on October 3, 2008

\title{
CYCLOTOMIC UNITS IN FUNCTION FIELDS
}

\author{
SUNGHAN BAE AND LINSHENG YIN \\ (Communicated by Wen-Ching Winnie Li)
}

\begin{abstract}
Let $k$ be a global function field over the finite field $\mathbb{F}_{q}$ with a fixed place $\infty$ of degree 1 . Let $K$ be a cyclic extension of degree dividing $q-1$, in which $\infty$ is totally ramified. For a certain abelian extension $L$ of $k$ containing $K$, there are two notions of the group of cyclotomic units arising from sign normalized rank 1 Drinfeld modules on $k$ and on $K$. In this article we compare these two groups of cyclotomic units.
\end{abstract}

\section{INTRODUCTION}

Let $K$ be an imaginary quadratic number field and $L$ an abelian extension of $\mathbb{Q}$ containing $K$. There exist two subgroups of the group of units of $L$. One is the group of cyclotomic units of the extension $L / \mathbb{Q}$ and the other the group of elliptic units of the extension $L / K$. Both have finite indices in the full group of units of $L$, which are closely related to the class number of $L$. The relation between these two groups was studied by Gillard Gi and Kersey Ke. In fact, it is shown that some power of an elliptic unit is a cyclotomic unit.

In this article we consider the analogous problem in the function field setting. Let $k$ be a global function field over the finite field $\mathbb{F}_{q}$ with a fixed place $\infty$ of degree 1. Let $\ell$ be an integer dividing $q-1$. Let $K$ be a cyclic extension of $k$ of degree $\ell$ in which $\infty$ is totally ramified, and let $L$ be an abelian extension of both $k$ and $K$, such that $\infty$ splits completely in $L / K$. In $L$ there exist two notions of the group of cyclotomic units. One group is over $k$ and the other over $K$. The latter can be viewed as an analogue of the group of elliptic units ([ABJ], YYi1, $\mathrm{Ou}$ ). We will compare these two groups, adopting the method of Gi].

We note that in Gi] there are some misprints. In the statement of Theorem 3, $12 f e(\mathfrak{f})$ should be changed to $12 \mathrm{eh}$. The reason for this is that the wrong formula was used (5 bis) on p. 187; it should be (see [GR], Proposition 7.19, or [0u, (3.3))

$$
L^{\prime}(0, \chi, K / k)=-\frac{1}{6 e h} \sum_{c \in C l((1))} \chi(c) \log |\delta(c)| .
$$

Received by the editors February 16, 2007.

2000 Mathematics Subject Classification. Primary 11R58.

The first author was supported by KOSEF research grants R01-2006-000-10320-0, F01-2006000-10040-0 and SRC program (ASARC R11-2007-035-01001-0).

The second author was supported by NSFC (No. 10571097).

(C)2008 American Mathematical Society Reverts to public domain 28 years from publication 
Notation. $k$ : a global function field over the finite field $\mathbb{F}_{q}$ of $q$ elements

$\infty$ : a fixed place of degree 1 of $k$

$\mathbb{A}$ : the ring of functions in $k$, which are regular away from $\infty$

$\ell$ : an integer dividing $q-1$

$K:=k\left(m^{1 / \ell}\right)$, where $m \in \mathbb{A}$ has degree prime to $\ell$

$\chi_{K}:=$ a fixed generator of the character group of $\operatorname{Gal}(K / k)$

It is clear that $\infty$ is totally ramified in $K / k$, so we use the same $\infty$ to denote the unique place of $K$ lying over $\infty$.

$\mathbb{B}$ : the integral closure of $\mathbb{A}$ in $K$, which is the same as the ring of functions in $K$ regular away from $\infty$

$\mathfrak{a}, \mathfrak{b}, \mathfrak{m}, \mathfrak{n}, \mathfrak{f}, \cdots:$ ideals of $\mathbb{A}$

$\mathfrak{A}, \mathfrak{B}, \mathfrak{M}, \mathfrak{N}, \mathfrak{F}, \cdots$ : ideals of $\mathbb{B}$

$h_{k}\left(\right.$ resp. $\left.h_{K}\right)$ : the class number of $k$ (resp. $K$ ), which is the same as the ideal class number of $\mathbb{A}($ resp. $\mathbb{B})$ since $\infty$ has degree 1

Fix a sign function $s g n: k_{\infty}=K_{\infty} \longrightarrow \mathbb{F}_{q}$ with $\operatorname{sgn}(0)=0$.

$k_{\mathfrak{n}}$ : the cylotomic function field over $k$ of conductor $\mathfrak{n}$ with respect to $s g n$

$K_{\mathfrak{N}}$ : the cylotomic function field over $K$ of conductor $\mathfrak{N}$ with respect to sgn

$G_{\mathfrak{n}}:=\operatorname{Gal}\left(k_{\mathfrak{n}} / k\right)$ and $\Gamma_{\mathfrak{N}}:=\operatorname{Gal}\left(K_{\mathfrak{N}} / K\right)$

$k_{(1)}\left(\right.$ resp. $\left.K_{(1)}\right)$ : the Hilbert class field of $k$ (resp. $K$ )

We choose the sign of $m$ so that $K$ is contained in $k_{(m)}$.

$\xi(\mathfrak{n})($ resp. $\xi(\mathfrak{N})): \xi$-invariant associated to $\mathfrak{n}$ (resp. $\mathfrak{N}$ )

$e_{\mathfrak{n}}\left(\right.$ resp. $\left.e_{\mathfrak{N}}\right)$ : the lattice function associated to the ideal $\mathfrak{n}$ (resp. $\mathfrak{N}$ )

For $\mathfrak{n} \neq(1)$ and $\mathfrak{N} \neq(1), \lambda_{\mathfrak{n}}:=\xi(\mathfrak{n}) e_{\mathfrak{n}}(1), \Lambda_{\mathfrak{N}}:=\xi(\mathfrak{N}) e_{\mathfrak{N}}(1)$.

For details of this notation we refer to [Ha1, Yi1.

\section{Preparation and statement of main theorem}

Let $L$ be an abelian extension of $k$ which is contained in some cyclotomic function field over $k$ and suppose $\infty$ splits completely in $L / K$. Let $O_{L}$ be the integral closure of $\mathbb{A}$ in $L$. For each ideal class $c$ (resp. $C$ ) of $\mathbb{A}$ (resp. $\mathbb{B})$ containing an ideal $\mathfrak{a}$ (resp. $\mathfrak{A})$, let

$$
\delta(c):=a \xi(\mathfrak{a})^{h_{k}} \quad \text { and } \quad \Delta(C):=A \xi(\mathfrak{A})^{h_{K}},
$$

where $(a)=\mathfrak{a}^{h_{k}}$ and $(A)=\mathfrak{A}^{h_{K}}$ with $\operatorname{sgn}(a)=\operatorname{sgn}(A)=1$.

For $\sigma \in G_{\mathfrak{n}}$, we define the partial zeta function by

$$
Z_{\mathfrak{n}}(s, \sigma):=\sum_{\sigma_{\mathfrak{b}}=\sigma,(\mathfrak{b}, \mathfrak{n})=(1)} N(\mathfrak{b})^{-s} .
$$

Note that $Z_{\mathfrak{n}}(0, \sigma)$ is a rational number. We return to $Z_{\mathfrak{n}}(s, \sigma)$ in the last section.

Let $\mathfrak{n}$ be the conductor of $L$ over $k$ and $\mathfrak{N}$ the conductor of $L$ over $K$, that is, $\mathfrak{n}\left(\right.$ resp. $\mathfrak{N}$ ) is the smallest ideal $\mathfrak{n}$ (resp. $\mathfrak{N}$ ) such that $L$ is contained in $k_{\mathfrak{n}}$ (resp. $\left.K_{\mathfrak{N}}\right)$. Let $\mathfrak{n}_{1}$ be the ideal $k \cap \mathfrak{N}$. Let $\Gamma:=\operatorname{Gal}(L / K)$ and $G:=\operatorname{Gal}(L / k)$. For $\mathfrak{n} \neq(1)$ and $g \in G($ resp. $\mathfrak{N} \neq(1)$ and $\gamma \in \Gamma)$, let

$$
\varphi_{L}(g):=\prod_{\tau \in G_{\mathfrak{n}},\left.\tau\right|_{L}=g} \lambda_{\mathfrak{n}}^{\tau}, \quad \Phi_{L}(\gamma)=\prod_{\tau \in \Gamma_{\mathfrak{N}},\left.\tau\right|_{L}=\gamma} \Lambda_{\mathfrak{N}}^{\tau} .
$$

For $\mathfrak{n}=(1)($ resp. $\mathfrak{N}=(1))$, we let

$$
\delta_{L}(g):=\prod_{\left.\sigma_{c}\right|_{L}=g} \delta(c), \quad \Delta_{L}(\gamma):=\prod_{\left.\sigma_{C}\right|_{L}=\gamma} \Delta(C),
$$


where $\sigma_{c}$ and $\sigma_{C}$ are the Artin automorphisms associated to $c$ and $C$, respectively.

Proposition 1 ([0u, Chap. 3, Chap. 4]). Let $g_{1}, g_{2}, g \in G$. Then we have

1) $\delta_{L}\left(g_{1}\right) / \delta_{L}\left(g_{2}\right)$ and $\varphi_{L}\left(g_{1}\right) / \varphi_{L}\left(g_{2}\right)$ are units in $O_{L}$.

2) $\left(\frac{\delta_{L}\left(g_{1}\right)}{\delta_{L}\left(g_{2}\right)}\right)^{g}=\frac{\delta_{L}\left(g g_{1}\right)}{\delta_{L}\left(g g_{2}\right)}$ and $\varphi_{L}\left(g_{1}\right)^{g}=\varphi_{L}\left(g g_{1}\right)$.

3) If $\mathfrak{n}$ is not a prime power, then $\varphi_{L}(g)$ is a unit in $O_{L}$.

The same holds for $\Delta, \Phi$ and $\Gamma$.

Let $P_{L / k}$ (resp. $P_{L / K}$ ) be the subgroup of $L^{*}$ generated by $\mathbb{F}_{q}^{*}, \varphi_{L}(g)$ and $\delta_{L}(g) / \delta_{L}(i d)$ for $g \in G$ (resp. $\Phi_{L}(\gamma)$ and $\Delta_{L}(\gamma) / \Delta_{L}(i d)$ for $\left.\gamma \in \Gamma\right)$, which we call the group of cyclotomic numbers over $k$ (resp. over $K$ ) in $L$. Let

$$
C_{L / k}:=P_{L / k} \cap O_{L}^{*}, \quad \text { and } \quad C_{L / K}:=P_{L / K} \cap O_{L}^{*},
$$

which we call the group of cyclotomic units of $L$ over $k$ and $K$, respectively. These are slightly different from the group of cyclotomic units defined in [ABJ] or [Yi1].

Let $S$ be the set of all prime ideals of $\mathbb{A}$, which are ramified in $L / k$ but unramified in $L / K$. For $\mathfrak{p} \in S$ denote by $T_{\mathfrak{p}}$ the inertia group in $L / k$ at $\mathfrak{p}$. Decompose $S$ into a disjoint union $S=\bigcup_{i \in I} S_{i}$, where two ideals in $S$ lie in the same $S_{i}$ if and only if they have the same inertia groups. Let $J$ be a subset of $I$ and $J_{1}$ its complementary subset. Let $L_{J}^{0}$ (resp. $L_{J}^{1}$ ) be the subfield of $L$ fixed by the subgroup $T_{J} \subset G$ (resp. $T_{J_{1}}$ ) generated by $T_{\mathfrak{p}}$ for any $\mathfrak{p} \in S_{i}$ with $i \in J$ (resp. $i \in J_{1}$ ). Let $\mathfrak{f}_{J}^{0}\left(\right.$ resp. $\mathfrak{f}_{J}$ ) be the conductor of $L_{J}^{0}$ (resp. $L_{J}^{1}$ ) over $k$. Let

$$
\theta_{J}:= \begin{cases}N_{k_{f_{J}^{0}} / L_{J}^{0}}\left(\lambda_{\mathfrak{f}_{J}^{0}}\right) & \text { if } \mathfrak{f}_{J}^{0} \neq(1), \\ N_{k_{(1)} / L_{J}^{0}} \delta(1) & \text { if } \mathfrak{f}_{J}^{0}=(1),\end{cases}
$$

and for $i=1, \ldots, \ell-1$,

$$
\beta_{J}^{(i)}(\sigma):=\chi_{K}^{i}(\sigma) \sum_{\tau \in G_{\mathfrak{f}_{J}^{1}},\left.\tau\right|_{L_{J}^{1}}=\sigma} Z_{\mathfrak{f}_{J}^{1}}(0, \tau),
$$

where $\sigma \in \operatorname{Gal}(L / k)$ and $\mathfrak{f}_{J}^{1}$ is the least common multiple of $\mathfrak{n}_{1}$ and $\mathfrak{f}_{J}$.

Let

$$
\begin{gathered}
m_{J}:=\frac{\left[L: L_{I}^{0}\right]^{2}}{\left[L: L_{J}^{0}\right]\left[L: L_{J}^{1}\right]}, \\
\beta_{J}^{(i)}:=\sum_{\sigma \in G} \beta_{J}^{(i)}(\sigma) \sigma^{-1}, \quad \beta_{J}=\prod_{i=1}^{\ell-1} \beta_{J}^{(i)}
\end{gathered}
$$

and

$$
\gamma_{J}:=\prod_{\mathfrak{p} \mid \mathfrak{n}_{1}, \mathfrak{p} \nmid \mathfrak{f}_{J}^{0}}\left(1-\sigma_{\mathfrak{p}}^{-1}\right),
$$

where $\sigma_{\mathfrak{p}}$ is the Frobenius automorphism of $\mathfrak{p}$ in $L_{J}^{0} / k$. Let $\alpha_{J}:=\gamma_{J} \beta_{J}$. But $\beta_{J} \in \mathbb{Q}(\zeta)[G]$, where $\zeta$ is a primitive $\ell$-th root of 1 . To proceed we need the following lemma.

Lemma 2. $\beta_{J} \in \mathbb{Q}[G]$.

Proof. Let $\eta \in \operatorname{Gal}(\mathbb{Q}(\zeta) / \mathbb{Q})$. We extend the action of $\eta$ to $\mathbb{Q}(\zeta)[G]$ in an obvious way. Then it is not hard to see that $\eta$ fixes $\beta_{J}$, which implies that $\beta_{J} \in \mathbb{Q}[G]$. 
Theorem 3. If $L / K$ is ramified, then

$$
\Phi_{L}^{\left[L: L_{I}^{0}\right]^{2}}=\epsilon \prod_{J \subset I} \theta_{J}^{-\ell^{2} \alpha_{J} m_{J}}
$$

with $\epsilon \in \mathbb{F}_{q}^{*}$. If $L / K$ is unramified and $\sum_{\sigma \in G} n(\sigma) \sigma \in \mathbb{Z}[G]$ satisfies $\sum n(\sigma)=0$, then

$$
\prod_{\sigma \in G} \Delta_{L}(\sigma)^{n(\sigma)\left[L: L_{I}^{0}\right]^{2}}=\epsilon\left(\prod_{J \subset I} \theta_{J}^{-\ell^{2} r_{J} \alpha_{J} m_{J}}\right)^{\sum n(\sigma) \sigma},
$$

with $\epsilon \in \mathbb{F}_{q}^{*}$, where

$$
r_{J}= \begin{cases}\frac{h_{K}}{h_{k}} & \text { if } f_{J}^{0}=(1) \\ h_{K} & \text { if } f_{J}^{0} \neq(1) .\end{cases}
$$

Note that $h_{K}$ is divisible by $h_{k}$.

\section{Proof of the MAin theorem}

Let $v_{k}$ be the normalized valuation of $k$ at $\infty$, and $v_{K}$ be the normalized valuation of $K$ at $\infty$. Then $v_{K}(a)=\ell v_{k}(a)$ for $a \in k$. We choose an extension $v_{1}$ (resp. $v_{2}$ ) of $v_{k}$ (resp. $v_{K}$ ) to $\bar{k}=\bar{K}$ so that $v_{2}(\alpha)=\ell v_{1}(\alpha)$, which we also denote by $v_{k}$ (resp. $\left.v_{K}\right)$. Let $\chi$ be a character of $\Gamma$. Let $\chi_{0}, \ldots, \chi_{\ell-1}$ be the characters of $G$ extending $\chi$. Assume that $\chi_{0}$ is real, that is, $\chi_{0}$ is trivial on the inertia group at $\infty$, and $\chi_{i}=\chi_{K}^{i} \chi_{0}$.

Let

$$
\begin{aligned}
\Sigma_{J}^{0}(\chi):= & \left\{\begin{array}{l}
\frac{1}{\left[L: L_{J}^{0}\right]} \sum_{\sigma \in G} \chi_{0}(\sigma) v_{k}\left(\sigma\left(\theta_{J}^{\gamma_{J}}\right)\right) \text { if } \mathfrak{f}_{J}^{0} \neq(1) \\
\frac{1}{h_{k}\left[L: L_{J}^{0}\right]} \sum_{\sigma \in G} \chi_{0}(\sigma) v_{k}\left(\sigma\left(\theta_{J}^{\gamma_{J}}\right)\right) \text { if } \mathfrak{f}_{J}^{0}=(1)
\end{array}\right. \\
& \Sigma_{J}^{i}(\chi):=\frac{1}{\left[L: L_{J}^{1}\right]} \sum_{\sigma \in G} \chi_{0}(\sigma) \beta_{J}^{(i)}(\sigma)
\end{aligned}
$$

and

$$
\begin{gathered}
\Sigma(\chi):=\sum_{\sigma \in \Gamma} \chi(\sigma) v_{K}\left(\Phi_{L}(\sigma)\right) \quad \text { if } \mathfrak{N} \neq(1) \\
\Sigma(\chi):=\frac{1}{h_{K}} \sum_{\sigma \in \Gamma} \chi(\sigma) v_{K}\left(\Delta_{L}(\sigma)\right) \quad \text { if } \mathfrak{N}=(1) .
\end{gathered}
$$

To prove Theorem 3 , it suffices to show that

$$
\left[L: L_{I}^{0}\right]^{2} \Sigma(\chi)=\ell^{2} \sum_{J \subset I} \sum_{\sigma \in \Gamma} \chi(\sigma) v_{K}\left(\sigma\left(\theta_{J}^{\alpha_{J}}\right)\right)=\sum_{J \subset I} \sum_{\sigma \in G} \chi_{0}(\sigma) v_{k}\left(\sigma\left(\theta_{J}^{\alpha_{J}}\right)\right) ;
$$

that is, it suffices to show that

$$
\Sigma(\chi)=\sum_{J \subset I}\left(\prod_{i=0}^{\ell-1} \Sigma_{J}^{i}(\chi)\right)
$$

Considering $\chi$ as a character of $G\left(L_{J}^{0} / k\right)$ or $G\left(L_{J}^{1} / k\right)$ if possible, we have

$$
\Sigma_{J}^{0}(\chi)= \begin{cases}\prod_{\mathfrak{p} \mid \mathfrak{n}_{1} \mathfrak{p} \nmid \mathfrak{f}_{J}^{0}}\left(1-\chi_{0}(\mathfrak{p})\right) \sum_{\sigma \in G_{\mathfrak{f}_{J}^{0}}} \chi_{0}(\sigma) v_{k}\left(\sigma\left(\lambda_{\mathfrak{f}_{J}^{0}}\right)\right) & \text { if } \mathfrak{f}_{J}^{0} \neq(1) \\ \prod_{\mathfrak{p} \mid \mathfrak{n}_{1}}\left(1-\chi_{0}(\mathfrak{p})\right) \sum_{\sigma \in G_{(1)}} \chi_{0}(\sigma) v_{k}(\sigma(\delta(1))) & \text { if } \mathfrak{f}_{J}^{0}=(1)\end{cases}
$$


if $\chi_{0}$ is trivial on $G\left(L / L_{J}^{0}\right)$ and 0 otherwise, and

$$
\Sigma_{J}^{i}(\chi)=\sum_{\sigma \in G_{f_{J}^{1}}} \chi_{i}(\sigma) Z_{\mathfrak{f}_{J}^{1}}(0, \sigma)
$$

if $\chi_{i}$ is trivial on $G\left(L / L_{J}^{1}\right)$ and 0 otherwise.

Remark. For $\mathfrak{p} \in S$ let $t_{\mathfrak{p}}$ be a generator of $T_{\mathfrak{p}}$. Then

$$
\chi_{i}\left(t_{\mathfrak{p}}\right)=\chi_{0}\left(t_{\mathfrak{p}}\right) \chi_{K}^{i}\left(t_{\mathfrak{p}}\right)=\zeta^{i} \chi_{0}\left(t_{\mathfrak{p}}\right),
$$

since $\mathfrak{p}$ is ramified in $K / k$, where $\zeta \neq 1$ is an $\ell$ th root of 1 . Thus $\chi_{i}$ is trivial on $T_{\mathfrak{p}}$ for some $i>0$ if and only if $\chi_{0}$ is not. Thus $\prod_{i=0}^{\ell-1} \Sigma_{J}^{i}(\chi) \neq 0$ if and only if the union of $S_{i}$, for $i \in J$ is exactly the set of $\mathfrak{p} \in S$ such that $\chi_{0}\left(t_{\mathfrak{p}}\right)=1$. For each $\chi$ this can happen for a unique $J$.

Thus Theorem 3 is equivalent to

Proposition 4. Let $\chi$ be a character of $\Gamma$, nontrivial if $\mathfrak{N}=(1)$. For the subset $J$ of $I$ as above, we have

$$
\Sigma(\chi)=\prod_{i=0}^{\ell-1} \Sigma_{J}^{i}(\chi) .
$$

Let $\mathfrak{f}_{i}$ be the conductor of $\chi_{i}$ for $i=0,1, \ldots, \ell-1$, and let $\mathfrak{F}$ be the conductor of $\chi$ as a character over $K$. We have the following properties of $L$-series (cf. [Yi1, Yi2, $\mathrm{Ou}$ ):

$$
L_{K}(s, \chi)=\prod_{i=0}^{\ell-1} L_{k}\left(s, \chi_{i}\right) .
$$

For a nontrivial character $\chi$, we have

$$
\begin{gathered}
L_{k}\left(0, \chi_{0}\right)=\frac{1}{q-1} \sum_{\sigma \in G\left(k_{\mathfrak{f}_{0}} / k\right)} \chi_{0}(\sigma) v_{k}\left(\lambda_{\mathfrak{f}_{0}}^{\sigma}\right) \quad \text { if } \mathfrak{f}_{0} \neq(1), \\
L_{k}\left(0, \chi_{0}\right)=\frac{1}{h_{k}(q-1)} \sum_{\sigma \in G\left(k_{(1)} / k\right)} \chi_{0}(\sigma) v_{k}(\delta(\sigma)) \quad \text { if } \mathfrak{f}_{0}=(1), \\
L_{K}(0, \chi)=\frac{1}{q-1} \sum_{\sigma \in G\left(K_{\mathfrak{F}} / K\right)} \chi(\sigma) v_{K}\left(\lambda_{\mathfrak{F}}^{\sigma}\right) \quad \text { if } \mathfrak{F} \neq(1), \\
L_{K}(0, \chi)=\frac{1}{h_{K}(q-1)} \sum_{\sigma \in G\left(K_{(1)} / K\right)} \chi(\sigma) v_{K}(\Delta(\sigma)) \quad \text { if } \mathfrak{F}=(1), \\
L_{k}\left(0, \chi_{i}\right)=B_{\chi_{i}}:=\sum_{\sigma \in G\left(k_{\mathfrak{f}_{i}} / k\right)} \bar{\chi}_{i}(\sigma) Z_{\mathfrak{f}_{i}}(0, \sigma), \quad i=1, \ldots, \ell-1 .
\end{gathered}
$$

Suppose that $\chi$ is nontrivial. Then

$$
\Sigma(\chi)=(q-1) \prod_{\mathfrak{P} \mid \mathfrak{N}}\left(1-(\chi(\mathfrak{P})) L_{K}(0, \chi) .\right.
$$

Here $\chi(\mathfrak{P})$ is 0 if $\mathfrak{p}$ divides the conductor of $\chi$ and $\chi\left(\sigma_{\mathfrak{P}}\right)$ otherwise.

Note that, for $\mathfrak{g} \neq(1)$ and $\mathfrak{f}_{0}=(1)$,

$$
\sum_{\sigma \in G_{\mathfrak{g}}} \chi_{0}(\sigma) v_{\infty}\left(\lambda_{\mathfrak{g}}^{\sigma}\right)=\prod_{\mathfrak{p} \mid \mathfrak{g}}\left(1-\chi_{0}(\mathfrak{p})\right) L_{k}\left(0, \chi_{0}\right) .
$$


Then

$$
\Sigma_{J}^{0}(\chi)=(q-1) \prod_{\mathfrak{p} \mid \mathfrak{n}_{1}}\left(1-\chi_{0}(\mathfrak{p})\right) L_{k}(0, \chi)
$$

and

$$
\Sigma_{J}^{i}=\prod_{\mathfrak{p} \mid \mathfrak{n}_{1}}\left(1-\chi_{i}(\mathfrak{p})\right) L_{k}\left(0, \chi_{i}\right), \quad i=1, \ldots, \ell-1 .
$$

Using the fact that

$$
\prod_{\mathfrak{P} \mid \mathfrak{N}}(1-\chi(\mathfrak{P}))=\prod_{\mathfrak{p} \mid \mathfrak{n}_{1}} \prod_{i=0}^{\ell-1}\left(1-\chi_{i}(\mathfrak{p})\right)
$$

we get

$$
\Sigma(\chi)=\prod_{i=0}^{\ell-1} \Sigma_{J}^{i}(\chi)
$$

Now assume that $\chi$ is trivial and $\mathfrak{N} \neq(1)$. Then $J=I$ in this case.

Case 1: $\mathfrak{n}_{1}$ contains at least two prime divisors.

Then so does $\mathfrak{N}$. Hence $\Phi_{K}$ and $\theta_{I}$ are units. Thus $\Sigma(\chi)=0=\Sigma_{I}^{0}(\chi)$.

Suppose that $\mathfrak{n}_{1}$ is a power of a prime $\mathfrak{p}$. Let $e, f, r$ be the ramification index, inertia degree and the number of primes over $\mathfrak{p}$ in $K$, respectively.

Case 2. $\mathfrak{n}_{1}$ is a power of a prime $\mathfrak{p}$ and $r>1$.

Then $\mathfrak{N}$ is not a prime power. So $\Sigma(\chi)=0$. On the other hand, $\Sigma_{I}^{i}(\chi)$ contains the factor $\left(1-\chi_{K}^{i}(\mathfrak{p})\right)$, which is 0 if $f \mid i$ and $e=1$.

Case 3. $\mathfrak{n}_{1}$ is a power of $\mathfrak{p}$ and $r=1$.

Let $\mathfrak{P}$ be the prime ideal of $K$ lying over $\mathfrak{p}$. Then one can show that (cf. [Ha2], $(2.3))$

$$
\begin{gathered}
\Sigma(\chi)=h_{K} \operatorname{deg} \mathfrak{P}=f h_{K} \operatorname{deg} \mathfrak{p}, \\
\Sigma_{I}^{0}(\chi)=h_{k} \operatorname{deg} \mathfrak{p}
\end{gathered}
$$

and

$$
\prod_{i=1}^{\ell-1} \Sigma_{I}^{i}(\chi)=\left(\prod_{i=1}^{\ell-1}\left(1-\chi_{K}^{i}(\mathfrak{p})\right)\right) \frac{h_{K}}{h_{k}}=f \frac{h_{K}}{h_{k}},
$$

since

$$
\chi_{K}^{i}(\mathfrak{p})= \begin{cases}0 & \text { if } e>1 \\ \zeta_{f}^{i} & \text { if } e=1,\end{cases}
$$

where $\zeta_{f}$ is a primitive $f$-th root of 1 . Hence we get the result in this case too.

\section{INTEGRALITY OF EXPONENTS}

Now the question is to know whether $\ell^{2} m_{J} \alpha_{J}$ is an element of $\mathbb{Z}[G]$. We want to determine $\beta_{J}^{(i)}(\sigma)$. For this we need more information about $Z_{\mathfrak{m}}(0, \sigma)$ for an ideal $\mathfrak{m}$ and $\sigma \in G$. It is well-known that $(\mathbb{A} / \mathfrak{m})^{*} \simeq \operatorname{Gal}\left(k_{\mathfrak{m}} / k_{\mathfrak{e}}\right) \subset \operatorname{Gal}\left(k_{\mathfrak{m}} / k\right)$. Let $X$ be the image of $\mathbb{F}_{q}^{*}$ under this isomorphism. $X$ is called the sign group of $G\left(k_{\mathfrak{m}} / k\right)$. Shu [Sh] constructed a set $G_{\mathfrak{m}}^{\prime}$ of coset representatives of $G_{\mathfrak{m}} / X$ and called the elements of $G_{\mathfrak{m}}^{\prime}$ monic. The following is due to Shu [Sh.

Proposition 5. Let $\mathfrak{m}$ be an ideal of $\mathbb{A}$ and $\sigma \in G_{\mathfrak{m}}$. 
a) The partial zeta function $Z_{\mathfrak{m}}(s, \sigma)$ is a rational function in $q^{-s}$ and $\left(1-q^{1-s}\right) Z_{\mathfrak{m}}(s, \sigma)$ is a polynomial in $q^{-s}$ with integer coefficients.

b) For any $a \neq i d \in X$ and any $\sigma \in G_{\mathfrak{m}}^{\prime}$, we have

$$
Z_{\mathfrak{m}}(s, \sigma)-Z_{\mathfrak{m}}(s, a \sigma)=q^{n(\sigma)} q^{-s j(\sigma)},
$$

for some appropriate nonnegative integers $n(\sigma)$ and $j(\sigma)$.

Let

$$
Y=\left\{a \in X: \chi_{K}(a)=1\right\} .
$$

Corollary 6. For any $\sigma \in G_{\mathfrak{m}}, \sum_{a \in Y} Z_{\mathfrak{m}}(0, a \sigma)$ is either $q^{n(\sigma)}+\frac{D}{\ell}$ or $\frac{D}{\ell}$ for some integer D.

Proof. There exist integers $C$ and $D$ such that $Z_{\mathfrak{m}}(0, \sigma)$ equals $\frac{C}{q-1}$ if $\sigma \in G_{\mathfrak{m}}^{\prime}$ and $\frac{D}{q-1}$ otherwise. From Proposition $\left.5 \mathrm{~b}\right), \frac{C}{q-1}-\frac{D}{q-1}=q^{n(\sigma)}$. Then the sum will be either

$$
\frac{C}{q-1}+\left(\frac{q-1}{\ell}-1\right) \frac{D}{q-1}=q^{n(\sigma)}+\frac{D}{\ell}
$$

or

$$
\frac{D}{q-1} \frac{q-1}{\ell}=\frac{D}{\ell}
$$

Write a set of representatives of the quotient group $G_{\mathfrak{f}_{J}^{1}} / Y$ by $W$. Then

$$
\sum_{\tau \in G_{f_{J}^{1}},\left.\tau\right|_{L_{J}^{1}}=\sigma} Z_{\mathfrak{f}_{J}^{1}}(0, \tau)=\sum_{\tau \in W,\left.\tau\right|_{L_{J}^{1}}=\sigma}\left(\sum_{a \in Y} Z_{\mathfrak{f}_{J}^{1}}(0, a \tau)\right) .
$$

Now letting $\mathfrak{m}=\mathfrak{f}_{J}^{1}$ in Corollary 6 , we see that the sum in the parentheses is either $q^{n(\tau)}+\frac{D}{\ell}$ or $\frac{D}{\ell}$ for some integer $D$. Thus $\ell \beta_{J}^{(i)} \in \mathbb{Z}[\zeta][G]$ and $\ell^{\ell-1} \beta_{J} \in \mathbb{Z}[G]$. Therefore $\Phi_{L}^{\ell^{\ell-3}\left[L: L_{I}\right]^{2}}$ is a cyclotomic number over $k$.

\section{REFERENCES}

[ABJ] Ahn, J., Bae, S., and Jung, H., Cyclotomic units and Stickelberger ideals of global function fields, Trans. AMS 355 (2003), 1803-1818. MR1953526 (2004m:11190)

[Gi] Gillard, R., Unités elliptiques et unités cyclotomiques, Math. Ann. 243 (1979), 181-189. MR543728 (81k:12007)

[GR] Gross, B. and Rosen, M., Fourier series and the special values of L-functions, Advances in Math. 69 (1988), 1-31. MR937316 (90k:11150)

[Ha1] Hayes, D., Stickelberger elements in function fields, Compos. Math. 55 (1985), 209-239. MR.795715 (87d:11091)

[Ha2] Elliptic units in function fields, Progress in Math. 26, Birkhäuser, Boston (1982), 321-340. MR685307 (84f:12005)

[Ke] Kersey, D., Modular units inside cyclotomic units, Ann. Math. (2) 112 (1980), 361-380. MR.592295 (82h:12006)

[Ou] Oukhaba, H., Fonctions discriminant, formules pour le nombre de classes, et unités elliptiques; Le cas des corps de fonctions (associé à des courbes sur des corps finis), Thèse, Institut Fourier, Grenoble, 1991.

[Sh] Shu, L., Narrow ray class fields and partial zeta functions, preprint, unpublished. 
[Yi1] Yin, L., Index-class number formulas over global function fields, Compos. Math. 109 (1997), 49-66. MR1473605 (98h:11151)

[Yi2] Stickelberger ideals and relative class numbers in function fields, J. Number Theory 81 (2000), 162-169. MR.1743498 (2001d:11114)

Department of Mathematics, Korea Advanced Institute of Science and Technology, DAEJeOn 305-701, Republic of Korea

E-mail address: shbae@math.kaist.ac.kr

Department of Mathematical Sciences, Tsinghua University, Beijing 100084, People's Republic of China

E-mail address: lsyin@math.tsinghua.edu.cn 